\section{Evaluación del rendimiento y la calidad de la zanahoria (Daucus carota $L$.) en dos sistemas de producción orgánico y convencional.}

Fernando Cofre Santo ${ }^{1}$ y Rubén Darío Saltos Espín

${ }^{1}$ Universidad Estatal Amazónica, Ecuador fcofre@uea.edu.ec

\section{Evaluation of the performance and quality of carrot (Daucus carota L.) in two organic and conventional production systems.}

Recibido: $12 / 12 / 2017$

Aceptado: $20 / 04 / 2018$

Publicado: 29/06/2018

\title{
RESUMEN
}

El propósito de este trabajo fue evaluar el rendimiento y la calidad de la zanahoria (Daucus carota L.) en dos sistemas de producción orgánico y convencional. El experimento se realizó en la hacienda San Gabriel de la provincia de Cotopaxi. Se utilizó un diseño de bloques completos al azar; se evaluaron los siguientes tratamientos: $\mathrm{T} 1=25 \mathrm{t} / \mathrm{ha}$ abono orgánico (Sistema orgánico); $\mathrm{T} 2=92 \mathrm{~kg} / \mathrm{ha}$ de $\mathrm{N}-21 \mathrm{~kg} / \mathrm{ha}$ de $\mathrm{P}_{2} \mathrm{O}_{5}-79.5 \mathrm{~kg} / \mathrm{ha}$ de $\mathrm{Ca}$ (Sistema convencional); testigo (T3). Estos fueron replicados cuatro veces, obteniéndose un total de 12 unidades experimentales. Los resultados obtenidos fueron examinados estadísticamente mediante el análisis de varianza, y para comparar medias de los tratamientos, se utilizó la prueba Tukey al 5\% y la diferencia mínima significativa al 5\%. Durante el ciclo del cultivo se evaluaron variables agronómicas y financieras: altura de planta, incidencia de plagas, longitud de raíz, calidad, rendimiento y la relación Beneficio-Costo $(\mathrm{B} / \mathrm{C})$. Al medir las productividades, el sistema que presentó mayor rendimiento fue el sistema orgánico con 17.81 t/ha. En relación al parámetro de calidad medido, se encontró diferencias altamente significativas: el sistema orgánico presentó una coloración y brillo característico de la zanahoria frente al sistema convencional. Del análisis financiero se desprende que la mejor tasa $B / C$ correspondió al sistema orgánico con 4.30 .

PALABRAS CLAVE: calidad, rendimiento, sistema convencional, sistema orgánico.

\section{ABSTRACT}

The purpose of this work was to evaluate the yield and quality of carrots (Daucus carota L.) In two organic and conventional production systems. The experiment was carried out at the San Gabriel farm in the province of Cotopaxi. A randomized complete block design was used; the following treatments were evaluated: $\mathrm{T} 1=25 \mathrm{t} /$ ha organic fertilizer (Organic system); $\mathrm{T} 2=92 \mathrm{~kg} / \mathrm{ha}$ of $\mathrm{N}-21 \mathrm{~kg} / \mathrm{ha}$ of P2O5 - $79.5 \mathrm{~kg} /$ ha of Ca (Conventional system); control (T3). These were replicated four times, obtaining 12 experimental units. The results obtained were statistically examined by means of the Analysis of Variance, and to compare means of the treatments, the Tukey test was used at $5 \%$ and the minimum significant difference at $5 \%$. Agronomic and financial variables were evaluated during the crop cycle: plant height, incidence of pests, root length, quality, yield and the Benefit-Cost $(B / C)$ ratio. When measuring productivity, the system that presented the highest yield It was the organic system with $17.81 \mathrm{t} / \mathrm{ha}$. In relation to the measured quality parameter, highly significant differences were found: the organic system showed a characteristic color and brightness of the carrot compared to the conventional system. The financial analysis shows that the best $\mathrm{B} / \mathrm{C}$ rate corresponded to the organic system with 4.30 .

KEYWORDS: conventional system, organic systems, performance, quality. 


\section{INTRODUCCIÓN}

A través de los ensayos de campo se ha comprobado que los sistemas orgánicos tienen rendimientos relativamente altos con una excelente eficiencia de entrada-salida. En 1978 y durante siete años, se comparó mediante un experimento la misma secuencia de cultivo para el sistema orgánico y convencional. Sus resultados demostraron que las parcelas orgánicas produjeron el $80 \%$ de los rendimientos frente al convencional; el uso de fertilizantes y el uso de energía se redujo en un $34 \%$ y un 53\% respectivamente; y las aplicaciones de plaguicidas se redujeron en un $97 \%$ (Mäder et al, 2002).

El aumento del consumo de alimentos cultivados orgánicamente en los últimos años se asocia con el interés generalizado de los consumidores en la seguridad y calidad de los alimentos. En general, estos se perciben como más saludables y seguros que los convencionales. La producción orgánica brinda beneficios agronómicos y ambientales como la preservación de los recursos naturales y la reducción de la contaminación del aire, agua, suelo y alimentos (Domagała-Świątkiewicz \& Gastol, 2012; Herencia et al, 2011; Søltoft et al, 2010).

Del mismo modo, los alimentos orgánicos pueden tener otras ventajas, como la ingestión de más compuestos fenólicos y vitaminas, y menos nitratos y pesticidas (Lima \& Vianello, 2011). Además, contienen concentraciones más altas de metales traza nutricionalmente beneficiosos y concentraciones más bajas de metales pesados nocivos (Kelly \& Bateman, 2010).

Las zanahorias (Daucus carota L.) son una de las hortalizas más cultivadas y consumidas en todo el mundo. Son alimentos ricos en humedad con un contenido de $90 \mathrm{~g} / 100 \mathrm{~g}$ sobre una base húmeda. Es considerada una de las verduras más saludables debido a su valor nutritivo y beneficios para la salud, relacionados con sus propiedades antioxidantes, anticancerígenas, antianémicas, cicatrizantes y sedantes (Doymaz, 2004; Gamboa-Santos et al, 2012).

En nuestro país su cultivo ha experimentado un importante crecimiento en los últimos años tanto en superficie como en producción. La variedad amarilla se produce en las zonas del Ecuador, en las provincias de Pichincha, Cotopaxi, Tungurahua, Bolívar y Chimborazo. Esta última, la que más cultiva esta especie con una producción al año de 10.30 toneladas, el $36.62 \%$ de la producción total en el Ecuador, que es de 28.13 toneladas. La mayor parte de la producción de zanahoria es para consumo interno. Solo se exporta un 3.9\% correspondiente a la variedad conocida como Babycarrot (zanahoria bebé), tanto fresca como congelada (Aragundi y Plúa, 2011).

Hasta el momento, el crecimiento de la producción y el manejo de sus resultados no se realizan de manera racional, lo cual determina que Ecuador no pueda ser competitivo con productos hortícolas como la zanahoria amarilla en los canales de comercialización internacionales.

Dada la respuesta poco clara del cultivo a la adición de abonos orgánicos, actualmente no se ha definido con exactitud el nivel de fertilización adecuado que le permita lograr la máxima producción y calidad. Sin embargo, es importante resaltar que un manejo inadecuado de la fertilización sintética (sistema convencional), además de causar alteraciones negativas en las actividades fisiológicas de la planta y su rendimiento (Mehdi et al, 2001) degrada el ambiente, por lo que es necesario integrar métodos de manejo sostenibles para la preservación del mismo (Méndez y Viteri, 2007). Frente a la realidad que vive el sector agrario y al interés de generar información local resulta preponderante valorar nuevos enfoques de producción, por ello se planteó como objetivo del presente trabajo: evaluar el rendimiento y calidad de la zanahoria (Daucus carota L.) en los sistemas de producción orgánico y convencional. 


\section{MATERIALES Y MÉTODOS}

El experimento se realizó en la hacienda San Gabriel, del cantón Latacunga de la provincia de Cotopaxi, latitud: $0^{\circ} 55^{\prime} 16.13^{\prime \prime S}$, longitud: $78^{\circ} 37^{\prime} 37.31^{\prime \prime O}$ a una altitud de 2920 metros sobre el nivel del mar. Con una temperatura media anual de $15{ }^{\circ} \mathrm{C}$, y una precipitación media anual de $400 \mathrm{~mm}$. Suelo de origen volcánico (Zebrowski, 1992) franco arenoso con pH 7.0 (neutro).

La investigación se fundamenta en el método científico experimental, puesto que se prueban varios tratamientos; y el método científico deductivo a raíz de la deducción de los efectos de lo aplicado, vinculados a herramientas como: conceptos, definiciones, hipótesis, variables e indicadores que permiten demostrar lo planteado.

\section{Características de la unidad de estudio}

Se establecieron parcelas de $5 \times 5 \mathrm{~m}$ cada una, es decir de $25 \mathrm{~m}^{2}$, con una distancia de separación de $1 \mathrm{~m}$ entre ellas y los bloques. El total del área experimental fue de $475 \mathrm{~m}^{2}$. Para el sistema de producción orgánico se aplicó materia orgánica descompuesta a razón de $62.5 \mathrm{~kg}$ por parcela neta $\left(25 \mathrm{~m}^{2}\right) \circ 25 \mathrm{t} / \mathrm{ha}$ al momento de la siembra. En el convencional se estableció en función de las recomendaciones del análisis de suelos dadas por el INIAP: $92 \mathrm{~kg} / \mathrm{ha}$ de $\mathrm{N}-21 \mathrm{~kg} / \mathrm{ha}$ de $\mathrm{P}_{2} \mathrm{O}_{5}-79.5$ $\mathrm{kg} / \mathrm{ha}$ de Ca respectivamente; una vez mezclados se aplicó al fondo del surco el $50 \%$ y la otra mitad a los 45 días de la siembra en banda lateral e incorporada al suelo con la ayuda de una azada.

\section{Diseño experimental}

Se utilizó el DBCA con tres tratamientos con cuatro repeticiones, en total 12 parcelas investigativas. Se evaluaron los siguientes tratamientos: $\mathrm{T} 1=25 \mathrm{t} / \mathrm{ha}$ abono orgánico (Sistema orgánico); T2= $92 \mathrm{~kg} / \mathrm{ha}$ de $\mathrm{N}$ - 21 kg/ha de $\mathrm{P}_{2} \mathrm{O}_{5}-79,5 \mathrm{~kg} / \mathrm{ha}$ de $\mathrm{Ca}$ (Sistema convencional); testigo (T3); estos fueron replicados 4 veces, obteniéndose un total de 12 unidades experimentales.

\section{Análisis estadístico}

Los resultados obtenidos fueron analizados estadísticamente mediante análisis de varianza, y para comparar medias de los tratamientos se utilizó la prueba Tukey al 5\% y la DMS al 5\%. Para el análisis de los datos se utilizó el paquete estadístico computarizado SAS 2009.

\section{Análisis Económico}

Se realizó mediante el cálculo de la Relación B/C.

\section{Variables evaluadas}

\section{- Altura de las plantas}

Se midió la altura de plantas a los 80, 100 y 120 días, tomados desde el cuello de la raíz hasta el ápice de la hoja más alta, la unidad de medida fue en centímetros $(\mathrm{cm})$.

\section{- Incidencia de pulgón (Dysaphisfoeniculus)}

Se evaluó en porcentaje a los 55, 80, 100 y 120 días. Se determinó la incidencia de pulgones aplicando la siguiente fórmula:

$$
\% \text { Incidencia }=\frac{\text { Número de plantas infestadas }}{\text { Número de plantas totales }} \times 100
$$


- Incidencia de enfermedades

Se evaluó en porcentaje a los a los 55, 80, 100 y 120 días, mediante la utilización de la fórmula

$$
\% \text { Incidencia } \frac{\text { Número de plantas infectadas }}{\text { Número de plantas totales }} \times 100
$$

- Diámetro (Ancho de hombro y mitad de raíz)

Se midió el diámetro de la raíz en el hombro y en la mitad de la zanahoria después de la cosecha, la unidad de medida fue en $\mathrm{mm}$ y se utilizó un calibrador pie de rey.

- $\quad$ Longitud de raíz

Se midió después de la cosecha, utilizando un calibrador pie de rey, la unidad de medida fue en $\mathrm{mm}$

- Calidad

Se determinó por su coloración y brillo en la cosecha, utilizando la siguiente escala:

De 2.1-3 Naranja brillante.

Entre 1.1-2 Naranja.

De 0-1 Naranja opaca.

- Rendimiento

Se calculó en base al peso de la zanahoria cosechada por cada tratamiento y transformado en t/ha.

\section{RESULTADOS Y DISCUSIÓN}

- Altura de planta en cm. A los 55, 80, 100 y 120 días

Tabla 1. Resultado de los tratamientos en la altura de plantas los 55, 80, 100 y 120 días.

\begin{tabular}{cccc}
\hline & Tratamientos & \multicolumn{2}{c}{ Alturas (cm) } \\
\hline Código & Descripción & 100 Días & 120 Días \\
S1 & Sistema Orgánico & $39.60 \mathrm{a}$ & $40.71 \mathrm{a}$ \\
S2 & Sistema Convencional & $37.53 \mathrm{a}$ & $39.28 \mathrm{a}$ \\
S3 & Testigo & $32.98 \mathrm{~b}$ & $34.51 \mathrm{~b}$ \\
\hline
\end{tabular}

Nota. Los promedios seguidos por letras iguales, en una misma columna, son estadísticamente iguales al 5\%. Fuente: Elaborado por el autor.

Realizado el análisis de varianza para altura de plantas tuvo significación estadística al 1\% para tratamientos a los 100 y 120 días; para la comparación ortogonal CO1: S3 vs S1, S2. Los promedios de altura fueron $10.81 \mathrm{~cm} ; 18.46 \mathrm{~cm} ; 36.71 \mathrm{~cm}$ y $38.16 \mathrm{~cm}$ a los 55, 80, 100 y 120 días respectivamente. Contrario a estos datos, en los efectuados a los 55 y 80 días no hubo diferencias en las alturas estadísticamente.

Con la prueba de Tukey al $5 \%$ para tratamientos en la variable altura de planta (Tabla 1 ), se obtuvo dos rangos de significación. A los 100 el sistema orgánico obtuvo $39.60 \mathrm{~cm}$ y el sistema convencional con $37.53 \mathrm{~cm}$ en el primer rango, el testigo se encuentra en el segundo rango con $32.98 \mathrm{~cm}$. A los 120 días 
el sistema orgánico tiene $40.71 \mathrm{~cm}$ y el sistema convencional con $39.28 \mathrm{~cm}$; ubicándose en el primer rango y el testigo en el segundo rango con $34.51 \mathrm{~cm}$. Los resultados de la prueba señalan la importancia de la nutrición de los cultivos para un crecimiento y desarrollo adecuado.

Con la prueba de la DMS al $5 \%$ para la comparación ortogonal (CO1: S3 vs S1, S2) el testigo (S3) obtuvo $34.51 \mathrm{~cm}$ de altura vs el promedio de los tratamientos de los sistemas orgánico (S2) y convencional (S3) con $39.99 \mathrm{~cm}$. Estas diferencias se deben principalmente al aporte de nutrientes al suelo; la cantidad de nutrientes que brinda el suelo para la planta fue insuficiente para el normal crecimiento y desarrollo de la misma.

\section{- Incidencia de pulgón (Dysaphisfoeniculus)}

Tabla 2. Efecto de los tratamientos en la variable incidencia de pulgón.

\begin{tabular}{cccc}
\hline \multicolumn{3}{c}{ Tratamientos } \\
\hline Código & Descripción & \%Incidencia \\
& & Sistema Orgánico & $3.25 \mathrm{a}$ \\
& S1 & Sistema Convencional & $3.25 \mathrm{a}$ \\
& S2 & Testigo & $12.67 \mathrm{~b}$ \\
\hline
\end{tabular}

Nota. Los promedios seguidos por letras iguales, en una misma columna, son estadísticamente iguales al 5\%. Fuente: Elaborado por el autor.

Con el análisis de varianza para incidencia de pulgones, se obtuvo significación estadística entre tratamientos y la comparación ortogonal testigo vs sistema orgánico y sistema convencional (CO1:S3 vs S1, S2). Realizada la prueba de Tukey al $5 \%$ para tratamientos en la variable incidencia de plagas (pulgones), se tienen dos rangos de significación, en el primero se ubican los tratamientos orgánico y químico con una incidencia de $3.25 \%$ y en el segundo rango el testigo con una diferencia considerable con $12.67 \%$.

Con los resultados de la prueba se establece que los sistemas orgánico y convencional tuvieron iguales comportamientos; una adecuada nutrición de la planta es fundamental para que pueda generar resistencia al ataque de cualquier plaga o enfermedad, confirmado por Dabike (2011), una planta bien nutrida y equilibrada, es más resistente a un ataque de plagas y enfermedades que uno desbalanceado.

Realizado el DMS para la comparación S3 vs S1, S2 en la variable incidencia de pulgones se tiene dos rangos de significación, el testigo (S3) con 3.25\% de incidencia versus el promedio entre el sistema orgánico (S1) y el sistema convencional (S2) con $12.67 \%$. El testigo al no recibir ningún tipo de manejo no tuvo la suficiente nutrición por lo que las plantas quedaron expuestas al ataque de plagas en relación a los sistemas orgánicos y químicos por el aporte de nutrientes que recibieron. 
- Incidencia de enfermedades

Tabla 3. Resultado de los tratamientos en la variable incidencia de enfermedades.

\begin{tabular}{ccc}
\hline & Tratamientos & \\
\hline Código & Descripción & \% Incidencia \\
s3 & Testigo & $17,10 \mathrm{a}$ \\
s2 & Sistema Convencional & $11,18 \mathrm{~b}$ \\
s1 & Sistema Orgánico & $10,70 \mathrm{~b}$ \\
\hline
\end{tabular}

Nota. Los promedios seguidos por letras iguales, en una misma columna, son estadísticamente iguales al 5\%. Fuente: Elaborado por el autor.

Realizado el análisis de varianza para la variable incidencia de enfermedades se obtuvo diferencias altamente significativas para tratamientos y la comparación ortogonal CO1: S3 vs S1, S2.

Con la prueba de Tukey al 5\% para tratamientos en la variable incidencia de enfermedades se tiene dos rangos de significación, el testigo tuvo $17.10 \%$ con mayor incidencia mientras que los sistemas químico y orgánico tuvieron incidencias de $11.18 \%$ y $10.70 \%$ respectivamente. Un suelo con buena fertilidad permite una alimentación equilibrada, produce plantas sanas con un alto grado de resistencia a las plagas y enfermedades. Una mal nutrida está por lo general más predispuesta al ataque de enfermedades (Organización Panamericana de la Salud, 2003).

Con la DMS al $5 \%$ se comparó el testigo (S3) con $17.10 \%$ vs el promedio de los tratamientos del sistema orgánico (S1) y sistema convencional (S2) con 10.94\% de incidencia de enfermedades. Esta diferencia se debe al aporte de nutrientes al suelo.

- Longitud de la raíz

Tabla 4. Efecto de los tratamientos en la variable longitud de raíz.

\begin{tabular}{ccc}
\hline \multicolumn{3}{c}{ Tratamientos } \\
\hline Código & Descripción & Promedio en mm \\
S1 & Sistema Orgánico & $129.56 \mathrm{a}$ \\
S2 & Sistema Convencional & $125.05 \mathrm{a}$ \\
S3 & Testigo & $108.39 \mathrm{~b}$ \\
\hline
\end{tabular}

Nota. Los promedios seguidos por letras iguales, en una misma columna, son estadísticamente iguales al 5\%. Fuente: Elaborado por el autor.

Realizado el análisis de varianza para la variable longitud de la raíz se tiene significación estadística para tratamientos y la comparación ortogonal CO1:S3 vs S1, S2.

La prueba de Tukey al 5\% para tratamientos en la variable longitud de la raíz, arrojó dos rangos de significación, el sistema orgánico obtuvo $129.56 \mathrm{~mm}$ seguido por el sistema convencional con $125.05 \mathrm{~mm}$ ubicados en el primer rango, el testigo obtuvo $108.39 \mathrm{~mm}$ de longitud. Los resultados señalan que tanto el tratamiento orgánico como el convencional tuvieron similar comportamiento en 
cuanto a la longitud de raíz. Sin embargo, el sistema orgánico matemáticamente fue el de mejor tratamiento.

Con la prueba de DMS al 5\% se comparó el testigo (S3) con $108.39 \mathrm{~mm}$ vs el promedio de los tratamientos del sistema orgánico (S1) y sistema convencional (S2) con $127.31 \mathrm{~mm}$ de longitud de raíz. Las diferencias de longitud se deben principalmente al aporte de nutrientes al suelo, la cantidad de nutrientes que brinda el suelo fue insuficiente para la planta.

- Calidad de la raíz

Tabla 5: Resultado de los tratamientos en la variable calidad de la raíz.

\begin{tabular}{ccccc}
\hline Tratamientos & & \multicolumn{3}{c}{ Escala de color } \\
\hline & & Naranja brillante & Naranja & Naranja opaca \\
Código & Descripción & $\mathbf{2 . 1 - \mathbf { 3 }}$ & $\mathbf{1 . 1 - \mathbf { 2 }}$ & $\mathbf{0 - \mathbf { 1 }}$ \\
& & & & \\
S1 & Sistema Orgánico & $2.78 \mathrm{a}$ & & \\
S2 & Sistema Convencional & & $1.71 \mathrm{~b}$ & $0.53 \mathrm{c}$ \\
S3 & Testigo & & \\
\hline
\end{tabular}

Nota. Los promedios seguidos por letras iguales, en una misma columna, son estadísticamente iguales al 5\%. Fuente: Elaborado por el autor.

Realizado el análisis de varianza para la variable calidad de raíz se establece que existe diferencias altamente significativas entre tratamientos y comparaciones ortogonales S3 vs S1, S2 y S1 vs S2.

La prueba de Tukey al $5 \%$ para tratamientos en la variable calidad de la raíz, mostró que hay tres rangos de significación claramente definidos; en el primer rango se tiene al tratamiento orgánico que presentó una coloración naranja brillante intenso característico de la zanahoria, está ubicada en la escala de 23; en el segundo rango se encuentra el tratamiento químico que tuvo un color anaranjado pero con menos brillo intenso, en una escala de 1-2; y por último se tiene al testigo que presentó un color naranja opaco correspondiente a una escala 0-1. Los resultados demuestran el efecto de los tratamientos sobre la calidad de la zanahoria, la que se midió en intensidad de color de acuerdo a la escala.

Además, los cambios de color en los alimentos pueden afectar a la aceptabilidad global del producto de los consumidores (Pingret, Fabiano-Tixier \& Chemat, 2013). El sistema orgánico a través de la aplicación de abonos orgánicos presentó un efecto positivo sobre la calidad de la raíz de la zanahoria. Altieri (1997), citado por Saltos (2010), mencionan que la materia orgánica por su acción antibiótica inhibe el crecimiento de ciertos hongos y bacterias que afectan los tubérculos y plantas, aumentando su resistencia a las plagas y agentes patógenos. 
Tabla 6. Resultado de la comparación sistema orgánico y sistema convencional versus testigo.

\begin{tabular}{|c|c|c|c|c|}
\hline \multicolumn{2}{|c|}{ Tratamientos } & \multicolumn{3}{|c|}{ Escala de color } \\
\hline Código & Descripción & $\begin{array}{c}\text { Naranja brillante } \\
2.1-3\end{array}$ & $\begin{array}{c}\text { Naranja } \\
1.2-2\end{array}$ & $\begin{array}{c}\text { Naranja opaca } \\
0-1\end{array}$ \\
\hline S1 y S2 & $\begin{array}{c}\text { Sistema orgánico y sistema } \\
\text { convencional }\end{array}$ & $2.24 \mathrm{a}$ & -- & \\
\hline S3 & Testigo & & & $0.53 \mathrm{~b}$ \\
\hline
\end{tabular}

Nota. Los promedios seguidos por letras iguales, en una misma columna, son estadísticamente iguales al 5\%. Fuente: Elaborado por el autor.

Realizado la prueba DMS al 5\% para la comparación ortogonal: testigo (S3) vs sistema orgánico (S1), sistema convencional (S2) en la variable calidad de la raíz, se tienen dos rangos de significación, el promedio de los valores de orgánico y convencional comparado con el testigo presenta diferencias en el color, el testigo presenta un color anaranjado pálido mientas que los tratamientos convencional y orgánico tienen un color más intenso y brilloso (Tabla 5).

Tabla 7. Resultado de la comparación sistema orgánico versus sistema convencional.

\begin{tabular}{ccccc}
\hline \multicolumn{2}{c}{ Tratamientos } & \multicolumn{3}{c}{ Escala de color } \\
\hline \multirow{2}{*}{ Código } & Descripción & Naranja brillante & Naranja & Naranja opaca \\
& & $\mathbf{2 . 1 - 3}$ & $\mathbf{1 . 3}-\mathbf{2}$ & $\mathbf{0 - 1}$ \\
S1 & Sistema Orgánico & $2.78 \mathrm{a}$ & & \\
S2 & Sistema convencional & & $1.71 \mathrm{~b}$ \\
\hline
\end{tabular}

Nota. Los promedios seguidos por letras iguales, en una misma columna, son estadísticamente iguales al 5\%. Fuente: Elaborado por el autor.

Con la prueba DMS al 5 \% para la comparación sistema orgánico (S1) versus sistema convencional (S2) en la variable calidad de la raíz, se tienen dos rangos de significación, el tratamiento orgánico es de un color naranja brillante comparado con el tratamiento químico que presenta un color naranja. El sistema orgánico a través de la aplicación de abonos orgánicos presentó un efecto positivo sobre la intensidad de color ligado que según Hurtado (2013). Los componentes responsables del color de las zanahorias son los carotenoides, pigmentos naranjas sensibles al calor, oxígeno, luz y a las reacciones enzimáticas. Su concentración es un parámetro importante en la determinación de la calidad, pues es un factor determinante del color y de la calidad nutricional.

\section{- $\quad$ Rendimiento $t / h a$}

La respuesta de los tratamientos en cuanto a la variable rendimiento $t / h a$ es muy diferente. Se obtuvo significación estadística para tratamientos y comparación ortogonal S3 vs S1, S2.

Realizada la prueba de Tukey al $5 \%$ para rendimientos en la variable rendimiento, se tienen dos rangos de significación, el sistema orgánico con 17.81 t/ha, seguido por el sistema convencional con 16.61 t/ha, ubicados en el primer rango; en el segundo rango se encuentra el testigo con 12.54 t/ha. Los 
resultados del análisis estadístico establecen que los sistemas orgánico y convencional tienen similares rendimientos comparados con el testigo; el sistema orgánico preserva la naturaleza sin afectar los rendimientos que se alcanzan con el sistema convencional en el que se utilizan productos sintéticos que son nocivos para la salud y el ambiente (Figura 1).

Figura 1. Efecto de los tratamientos en el rendimiento $t / h a$.

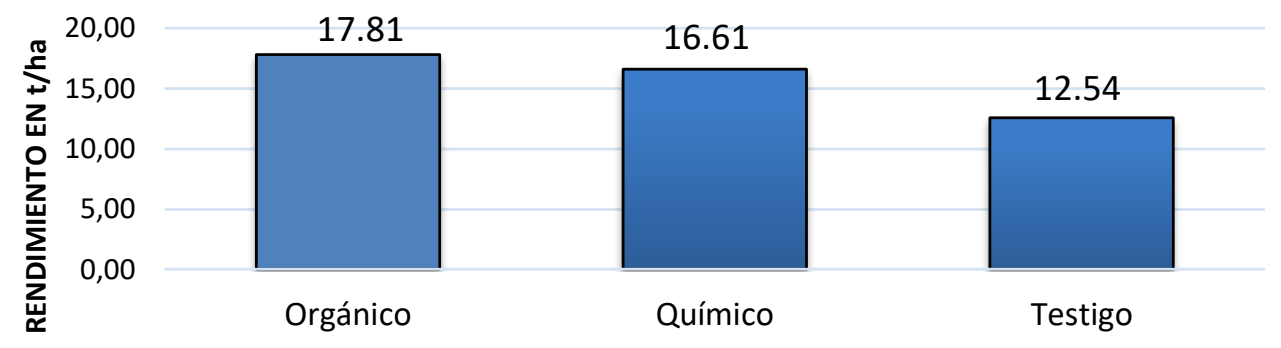

Fuente: Elaborado por el autor.

Con la prueba de DMS al $5 \%$ se comparó el testigo (s3) con $12.54 \mathrm{t} / \mathrm{ha}$ vs el promedio de los sistemas orgánico (S1) y convencional (S2) con $17.21 \mathrm{t} / \mathrm{ha}$. Las diferencias de rendimiento se deben principalmente al aporte de nutrientes al suelo; la cantidad de nutrientes que brinda el suelo a la planta fue insuficiente para la producción de zanahorias. El rendimiento que puede aportar un cultivo depende de sus características genéticas de productividad potencial, rusticidad y de las condiciones ambientales (Hernández, Soto y Plana, 2015).

- Análisis Económico

Tabla 8. Costos por tratamiento en dólares.

\begin{tabular}{lccccr}
\hline \multicolumn{1}{c}{ Tratamientos } & Costo fijo/Ha & $\begin{array}{c}\text { Costo } \\
\text { variable/Ha }\end{array}$ & $\begin{array}{c}\text { Costo } \\
\text { total }\end{array}$ & qq/Ha & Costo/qq \\
\hline Sistema orgánico & 817.55 & 545.04 & 1362.59 & 356.20 & 3.83 \\
Sistema convencional & 730.42 & 486.95 & 1217.37 & 332.20 & 3.66 \\
Testigo & 654.60 & 436.40 & 1091 & 250.80 & 4.35 \\
\hline
\end{tabular}

Fuente: Elaborado por el autor.

Los costos por tratamiento se determinaron sobre la base de los insumos, materiales, equipos y herramientas que se utilizaron en cada uno de los sistemas de producción, un costo para el sistema orgánico, otro costo para el sistema convencional y un tercero para el testigo; luego con el rendimiento producido en quintales por hectárea se calculó el costo de producción por quintal de zanahoria.

Tabla 9. Ingresos por tratamiento en dólares.

\begin{tabular}{lcccc}
\hline Tratamientos & $\begin{array}{c}\text { Rendimiento } \\
\text { t/ha }\end{array}$ & $\begin{array}{c}\text { Rendimiento } \\
\text { qq/Ha }\end{array}$ & $\begin{array}{c}\text { Ingreso } \\
\text { unitario/qq }\end{array}$ & $\begin{array}{c}\text { Ingreso } \\
\text { Total/qq }\end{array}$ \\
\hline Sistema orgánico & 17.81 & 356.2 & 8 & 2849.6 \\
Sistema convencional & 16.61 & 332.2 & 7 & 2325.4 \\
Testigo & 12.54 & 250.8 & 5 & 1254 \\
\hline
\end{tabular}

Fuente: Elaborado por el autor. 
Los ingresos por tratamiento se establecieron mediante el costo por cada quintal que en esa época se vendió.

Tabla 10. Cálculo de la rentabilidad.

\begin{tabular}{lcccc}
\hline \multicolumn{1}{c}{ Tratamiento } & Costo USD & Ingreso USD & Beneficio & Utilidad \\
\hline Sistema orgánico & 3.83 & 8.00 & 4.17 & 109.13 \\
Sistema convencional & 3.66 & 7.00 & 3.34 & 91.02 \\
Testigo & 4.35 & 5.00 & 0.65 & 14.94 \\
\hline
\end{tabular}

Fuente: Elaborado por el autor

El cálculo de la rentabilidad se realizó por cada quintal de zanahoria, para lo cual se establecieron los costos y los ingresos, con estos valores se determinó el beneficio que resulta de la resta del ingreso menos el costo, y por último se calculó la rentabilidad dividiendo el beneficio para el costo y transformándolo a porcentaje.

Desde el punto de vista económico, el tratamiento de mayor rentabilidad fue el sistema orgánico que tuvo una rentabilidad del $109.13 \%$, en segundo lugar, está el sistema convencional con $91.02 \%$ y por último el testigo, que tuvo una rentabilidad del $14.94 \%$.

\section{CONCLUSIONES}

Luego de haber realizado los diferentes análisis estadísticos y agronómicos se concluye lo siguiente:

Las etapas fenológicas del cultivo de la zanahoria como altura de plantas a los 55, 80, 100 y 120 días, diámetros y longitud de la raíz en las parcelas demostrativas tuvieron similar comportamiento, tanto en el sistema orgánico como convencional, pero sí hubo diferencias frente al testigo del mismo modo en cuanto la incidencia de plagas y enfermedades.

El rendimiento de los tratamientos fueron diferentes, el orgánico obtuvo $17.81 \mathrm{t} / \mathrm{ha}$, seguido del convencional con un rendimiento de $16.11 \mathrm{t} / \mathrm{ha}$, mientras que el testigo obtuvo $12.54 \mathrm{t} / \mathrm{ha}$. En cuanto a la calidad, la zanahoria orgánica tuvo mejor apariencia con un color naranja brillante característico que puede ser comercializado en mercados diferenciados a precio justo.

De los costos de producción el sistema orgánico fue el más costoso con 3.83 dólares por quintal, luego el convencional con 3.66 dólares por quintal. La mayor rentabilidad se alcanzó con el tratamiento orgánico que tuvo un $109.13 \%$.

Se recomienda a las instituciones públicas y privadas realizar procesos de transferencia de tecnología de los resultados obtenidos.

\section{REFERENCIAS BIBLIOGRÁFICAS}

Aragundi, K. y Plúa, B. (2011). Utilización de harina de zanahoria amarilla (Daucus carota) en la elaboración de pan. [Tesis de grado, Escuela Superior Politécnica del Litoral]. Repositorio Institucional. http://www.dspace.espol.edu.ec/xmlui/handle/123456789/17026 
Dabike, J. (2011). Chile: Una planta nutrida y equilibrada es más resistente a un ataque de plagas o enfermedades. [Comentario]. https://n9.cl/3801

Domagała-Świątkiewicz, I. \& Gastol, M. (2012). Comparative study on mineral content of organic and conventional carrot, celery and red beet juices. Acta scientiarum Polonorum. Hortorum cultus = Ogrodnictwo, 11(2), 173-183. http://www.hortorumcultus.actapol.net/pub/11 2 173.pdf

Doymaz, I. (2004). Convective air drying characteristics of thin layer carrots. Journal of Food Engineering, 61(3), 359-364. https://doi.org/10.1016/S0260-8774(03)00142-0

Gamboa-Santos, J., Montilla, A., Soria, A. C. y Villamiel, M. (2012). Effects of conventional and ultrasound blanching on enzyme inactivation and carbohydrate content of carrots. European Food Research and Technology, 234(6), 1071-1079. https://link.springer.com/article/10.1007/s00217-012-1726-7

Herencia, J. F., Garcia-Galavis, P. A., Dorado, J. A. R. \& Maqueda, C. (2011). Comparison of nutritional quality of the crops grown in an organic and conventional fertilized soil. Scientia Horticulturae, 129(4), 882-888. https://doi.org/10.1016/i.scienta.2011.04.008

Hernández, N., Soto, F. y Plana, R. (2015). Comportamiento del crecimiento y rendimiento del cultivo del trigo (Triticumaestivum L.) en tres fechas de siembra. Cultivos Tropicales, 36(1), 86-92. https://n9.cl/rhy7

Hiranvarachat, B., Devahastin, S. y Chiewchan, N. (2011). Effects of acid pretreatments on some physicochemical properties of carrot undergoing hot air drying. Food Bioprod. Process, 89(2) 116-127. https://doi.org/10.1016/j.fbp.2010.03.010

Hurtado, S. (2013). Efecto de la aplicación de ultrasonidos en Vegetales-Impacto sobre la Microbiota, Textura y color de la Zanahoria (Daucus carota) [tesis de Grado, Universitat Politécnica de Catalunya]. Repositorio Institucional. https://upcommons.upc.edu/handle/2099.1/20185

INFOAGRO. (2014). El Cultivo de Zanahoria. [Blog]. http//www.infoagro.com/hortalizas/zanahoria.htm

Kelly, S. D. \& Bateman, A. S. (2010). Comparison of mineral concentrations in commercially grown organic and conventional crops - Tomatoes (Lycopersicon esculentum) and lettuces (Lactuca sativa). Food Chemistry, 119(2), 738-745. https://doi.org/10.1016/j.foodchem.2009.07.022

Lima, G. P. P. \& Vianello, F. (2011). Review on the main differences between organic and conventional plant-based foods. International Journal of Food Science \& Technology, 46(1), 1-13. https://doi.org/10.1111/j.1365-2621.2010.02436.x

Lin, T.M., Durance, T.D. \& Scaman, C.H. (1998). Characterization of vacuum microwave, air and freeze dried carrot slices. Food Research International, 31(2) 111-117. https://doi.org/10.1016/S0963-9969(98)00070-2

Mäder, P., Fliessbach, A., Dubois, D., Gunst, L., Fried, P. y Niggli, U. (2002). Soil Fertility and Biodiversity in Organic Farming. Science, 296(5573), 1694-1697. https://doi.org/10.1126/ science.1071148

Mehdi, S. M.; Sahkir, A.; Sadiq, M., Sarfaraz, M., Hassan, G., Akhtar, J. \& Jarnil, M. (2001). Effect of phosphorus, zinc and farm yard manure in the presence of nitrogen and potash on NP and Zn concentration in rice. Pakistan Journal of Biological Sciences, 4(2), 342-343. http://docsdrive.com/pdfs/ansinet/pjbs/2001/156-158.pdf 
Méndez, M. J. y Viteri, S. E. (2007). Alternativas de biofertilización para la producción sostenible de cebolla de bulbo (Allium cepa) en Cucaita, Boyacá. Agronomía Colombiana, 25(1) 168-175. https://www.redalyc.org/pdf/1803/180316240019.pdf

Organización Panamericana de la Salud. (2003). Manejo de plagas sin químicos: manual para docentes. http://www.bvsde.paho.org/foro hispano/2 plagas.pdf

Pingret, D., Fabiano-Tixier, A. S. \& Chemat, F. (2013). Degradation during application of ultrasound in food processing: a review. Food control, 31(2), 593-606. https://doi.org/10.1016/j.foodcont.2012.11.039

Saltos, R. (2010). Efecto de la fertilización química y orgánica sobre el rendimiento y calidad de papa nativa dolores (Solanumsp) en la comunidad Marcopamba, provincia Bolívar. [tesis de grado, Universidad Estatal de Bolívar]. Repositorio Institucional. http://biblioteca.ueb.edu.ec/cgi$\underline{\text { bin/koha/opac-detail.pl?biblionumber }=17884}$

Søltoft, M., Bysted, A., Madsen, K. H., Mark, A. B., Bügel, S. G., Nielsen, J. \& Knuthsen, P. (2010). Effects of organic and conventional growth systems on the content of carotenoids in carrot roots, and on intake and plasma status of carotenoids in humans. Journal of the Science of Food and Agriculture, 91(4), 767-775. https://doi.org/10.1002/isfa.4248

Wu, B., Pan, Z., Qu, W., Wang, B., Wang, J. \& Ma, H. (2014). Effect of simultaneous infrared dryblanching and dehydration on quality characteristics of carrot slices. LWT-Food Science and Technology, 57(1), 90-98. https://doi.org/10.1016/i.Iwt.2013.11.035

Zebrowski, C. (1992). Los suelos volcánicos endurecidos en América Latina. Terra, 10 (especial). https://core.ac.uk/download/pdf/39860629.pdf 